\title{
Polyalkynes Capped by Sulfur and Selenium
}

\author{
Daniel B. Werz, Rolf Gleiter* \\ Organisch-Chemisches Institut der Universität Heidelberg, \\ Im Neuenheimer Feld 270, D-69120 Heidelberg, Germany. \\ e-mail: rolf.gleiter@urz.uni-heidelberg.de
}

\section{Supporting Information}

1.) General Experimental S2

2.) Computational Details S2

3.) Total Energies, Number of Imaginary Frequencies (NIMAG) and Cartesian Coordinates for $1(n), 2(n)$ and $3(n)(n=1-4) \quad S 2$

4.) $\mathrm{He}(\mathrm{II})$ Photoelectron Spectra of 2(2) and 3(2) S20

5.) X-ray Data and Details of the Refinement Procedure for 3(4) S21 


\section{1.) General Experimental, X-ray Diffraction Analyses and Photoelectron Spectroscopy. All}

reactions were conducted in oven-dried glassware under an argon atmosphere with magnetic stirring. THF was dried with sodium/benzophenone and distilled under argon before use. Melting points are uncorrected. Materials used for column chromatography: Silica gel 60 . ${ }^{1} \mathrm{H} N M R$ and ${ }^{13} \mathrm{C}$ NMR were recorded either at 300 and $500 \mathrm{MHz}\left({ }^{1} \mathrm{H} \mathrm{NMR}\right)$ or 75.5 and 125.8 $\mathrm{MHz}\left({ }^{13} \mathrm{C} N M R\right)$, respectively, using the solvent as internal standard. The IR spectra were recorded with a FT-IR instrument. The UV light absorption spectra were recorded using a FTUV spectrometer. The high-resolution mass spectra (HRMS) were recorded in the EI (70 eV) mode. Elemental analyses were carried out by the Mikroanalytisches Laboratorium der Universität Heidelberg.

The reflections were collected with a CCD diffractometer (Mo- $K_{\square}$ radiation, graphite monochromator). Intensities were corrected for Lorentz and polarization effects, and an empirical absorption correction was applied using SADABS based on the Laue symmetry of the reciprocal space. The structures were solved by direct methods. The structural parameters of the non-hydrogen atoms were refined anisotropically according to a full-matrix least-squares technique $\left(F^{2}\right)$. The hydrogen atoms were calculated according to stereochemical aspects. Structure solution and refinement were carried out with SHELXTL (5.10) software package.

The photoelectron spectra were recorded with a spectrometer that was equipped with a lamp giving both $\mathrm{He}(\mathrm{I})$ and $\mathrm{He}(\mathrm{II})$ radiation. Where a clear separation of bands was found, relative intensities were estimated by dividing the band areas. The spectra were recorded at least twice. The count rates for $\mathrm{He}$ (I) were $400 \mathrm{cps}$, for $\mathrm{He}$ (II) 180-200 cps. The spectra were calibrated with Ar and Xe. A resolution of $20 \mathrm{meV}$ was obtained for the ${ }^{2} \mathrm{P}_{3 / 2}$ line of Ar.

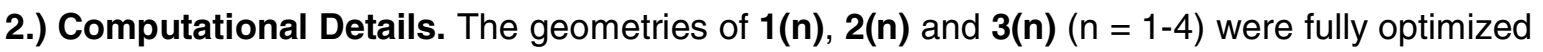
with GAUSSIAN98 at the density functional level of theory (B3LYP) using the split-valence 6-311G* basis set for $\mathrm{C}, \mathrm{H}, \mathrm{O}, \mathrm{S}$ and Se. Frequency calculations were carried out to characterize the nature of the stationary points. The electronic structures were analyzed by means of natural bond orbital (NBO) analyses. For all compounds a $C_{2}$ symmetric global minimum is revealed. The cartesian coordinates (in standard orientation), number of imaginary frequencies (NIMAG) and total energies (HF) of the different conformers are given in the following.

\section{3.) Total energies, number of imaginary frequencies (NIMAG) and cartesian coordinates} for $1(n), 2(n)$ and $3(n)(n=1-4)$.

- $1(1)\left(C_{2}\right)$ 


\begin{tabular}{|c|c|c|c|c|c|}
\hline \multicolumn{6}{|c|}{$H F=-306.4296087$} \\
\hline \multicolumn{6}{|c|}{ NIMAG $=0$} \\
\hline \multirow{2}{*}{$\begin{array}{l}\text { Center } \\
\text { Number }\end{array}$} & \multirow{2}{*}{$\begin{array}{l}\text { Atomic } \\
\text { Number }\end{array}$} & \multirow{2}{*}{$\begin{array}{l}\text { Atomic } \\
\text { Type }\end{array}$} & \multicolumn{3}{|c|}{ Coordinates (Angstroms) } \\
\hline & & & $x$ & $\mathrm{Y}$ & Z \\
\hline 1 & 8 & 0 & -.402023 & 1.866492 & -.377011 \\
\hline 2 & 6 & 0 & -.120807 & .587449 & -.340586 \\
\hline 3 & 6 & 0 & .402023 & 2.687246 & .493011 \\
\hline 4 & 1 & 0 & .048819 & 3.706816 & .350353 \\
\hline 5 & 1 & 0 & 1.456445 & 2.610060 & .217663 \\
\hline 6 & 1 & 0 & .266742 & 2.381972 & 1.533518 \\
\hline 7 & 6 & 0 & .120807 & -.587449 & -.340586 \\
\hline 8 & 8 & 0 & .402023 & -1.866492 & -.377011 \\
\hline 9 & 6 & 0 & -.402023 & -2.687246 & .493011 \\
\hline 10 & 1 & 0 & -.048819 & -3.706816 & .350353 \\
\hline 11 & 1 & 0 & -1.456445 & -2.610060 & 0 \\
\hline 12 & 1 & 0 & -.266742 & -2.381972 & $2 \quad 1.533518$ \\
\hline
\end{tabular}

- $\quad 1(1)\left(C_{2 h}\right)$

$H F=-306.4272726$

$\mathrm{NIMAG}=1$

\begin{tabular}{|c|c|c|c|c|c|}
\hline \multirow{2}{*}{$\begin{array}{l}\text { Center } \\
\text { Number }\end{array}$} & \multirow{2}{*}{\multicolumn{2}{|c|}{$\begin{array}{l}\text { Atomic } \\
\text { Number }\end{array}$}} & \multirow{2}{*}{$\begin{array}{l}\text { Atomic } \\
\text { Type }\end{array}$} & \multicolumn{2}{|c|}{ Coordinates (Angstroms } \\
\hline & & & & $x$ & Z \\
\hline 1 & 8 & 0 & .005898 & 1.912978 & .000000 \\
\hline 2 & 6 & 0 & -.005898 & .598916 & .000000 \\
\hline 3 & 6 & 0 & -1.298351 & 2.520034 & .000000 \\
\hline 4 & 1 & 0 & -1.125575 & 3.594467 & .000000 \\
\hline 5 & 1 & 0 & -1.854568 & 2.226856 & .894152 \\
\hline 6 & 1 & 0 & -1.854568 & 2.226856 & -.894152 \\
\hline 7 & 6 & 0 & .005898 & -.598916 & .000000 \\
\hline
\end{tabular}




$\begin{array}{cccccc}8 & 8 & 0 & -.005898 & -1.912978 & .000000 \\ 9 & 6 & 0 & 1.298351 & -2.520034 & .000000 \\ 10 & 1 & 0 & 1.125575 & -3.594467 & .000000 \\ 11 & 1 & 0 & 1.854568 & -2.226856 & .894152 \\ 12 & 1 & 0 & 1.854568 & -2.226856 & -.894152\end{array}$

- $1(2)\left(C_{2}\right)$

$\mathrm{HF}=-382.6121423$

$\mathrm{NIMAG}=0$

\begin{tabular}{|c|c|c|c|c|c|}
\hline \multirow{2}{*}{$\begin{array}{l}\text { Center } \\
\text { Number }\end{array}$} & \multirow{2}{*}{\multicolumn{2}{|c|}{$\begin{array}{l}\text { Atomic } \\
\text { Number }\end{array}$}} & \multirow{2}{*}{$\begin{array}{l}\text { Atomic } \\
\text { Type }\end{array}$} & \multicolumn{2}{|c|}{ Coordinates (Angstroms) } \\
\hline & & & & $\mathrm{Y}$ & Z \\
\hline 1 & 8 & 0 & -.060568 & -.380200 & -3.178531 \\
\hline 2 & 6 & 0 & -.086326 & $-.303374 \quad-$ & -1.884508 \\
\hline 3 & 6 & 0 & 1.257869 & -.448004 & -3.772891 \\
\hline 4 & 1 & 0 & 1.092456 & -.508660 & -4.846066 \\
\hline 5 & 1 & 0 & 1.781633 & -1.336338 & -3.415427 \\
\hline 6 & 1 & 0 & 1.825150 & .450447 & -3.523086 \\
\hline 7 & 6 & 0 & -.152381 & -.230875 & -.678883 \\
\hline 8 & 8 & 0 & -.380200 & -.060568 & 3.178531 \\
\hline 9 & 6 & 0 & -.448004 & 1.257869 & 3.772891 \\
\hline 10 & 1 & 0 & -.508660 & 1.092456 & 4.846066 \\
\hline 11 & 1 & 0 & -1.336338 & 1.781633 & 3.415427 \\
\hline 12 & 1 & 0 & .450447 & 1.825150 & 3.523086 \\
\hline 13 & 6 & 0 & -.230875 & -.152381 & .678883 \\
\hline 14 & 6 & 0 & -.303374 & -.086326 & 1.884508 \\
\hline
\end{tabular}

- $\quad 1(2)\left(C_{2 \mathrm{~h}}\right)$

$H F=-382.6108769$

$\mathrm{NIMAG}=1$ 


\begin{tabular}{|c|c|c|c|c|c|}
\hline \multirow{2}{*}{$\begin{array}{l}\text { Center } \\
\text { Number }\end{array}$} & \multirow{2}{*}{\multicolumn{2}{|c|}{$\begin{array}{l}\text { Atomic } \\
\text { Number }\end{array}$}} & \multirow{2}{*}{$\begin{array}{l}\text { Atomic } \\
\text { Type }\end{array}$} & \multicolumn{2}{|c|}{ Coordinates (Angstroms) } \\
\hline & & & & $x$ & Z \\
\hline 1 & 8 & 0 & .315849 & .000000 & -3.173249 \\
\hline 2 & 6 & 0 & .214182 & .000000 & -1.878741 \\
\hline 3 & 6 & 0 & 1.667214 & .000000 & -3.689051 \\
\hline 4 & 1 & 0 & 1.566978 & .000000 & -4.771881 \\
\hline 5 & 1 & 0 & 2.194495 & -.895221 & -3.353833 \\
\hline 6 & 1 & 0 & 2.194495 & .895221 & -3.353833 \\
\hline 7 & 6 & 0 & .079485 & .000000 & -.677713 \\
\hline 8 & 8 & 0 & -.315849 & .000000 & 3.173249 \\
\hline 9 & 6 & 0 & -1.667214 & .000000 & 3.689051 \\
\hline 10 & 1 & 0 & -1.566978 & .000000 & 4.771881 \\
\hline 11 & 1 & 0 & -2.194495 & -.895221 & 3.353833 \\
\hline 12 & 1 & 0 & -2.194495 & .895221 & 3.353833 \\
\hline 13 & 6 & 0 & -.079485 & .000000 & .677713 \\
\hline 14 & 6 & 0 & -.214182 & .000000 & 1.878741 \\
\hline
\end{tabular}

- $1(3)\left(C_{2}\right)$

$H F=-458.7921365$

$\mathrm{NIMAG}=0$

\begin{tabular}{|c|c|c|c|c|c|}
\hline \multirow{2}{*}{$\begin{array}{l}\text { Center } \\
\text { Number }\end{array}$} & \multirow{2}{*}{\multicolumn{2}{|c|}{$\begin{array}{l}\text { Atomic } \\
\text { Number }\end{array}$}} & Atomic & \multicolumn{2}{|c|}{ Coordinates (Angstron } \\
\hline & & & Type & $x$ & Z \\
\hline 1 & 8 & 0 & -.033507 & 4.466802 & -.265767 \\
\hline 2 & 6 & 0 & -.001361 & 3.175905 & -.231748 \\
\hline 3 & 6 & 0 & .888205 & 5.145569 & .625819 \\
\hline 4 & 1 & 0 & .721271 & 6.207231 & .461947 \\
\hline 5 & 1 & 0 & 1.914112 & 4.873898 & .372854 \\
\hline 6 & 1 & 0 & .667438 & 4.876478 & 1.659845 \\
\hline 7 & 6 & 0 & .000664 & 1.963710 & -.226800 \\
\hline
\end{tabular}




$\begin{array}{cccccc}8 & 8 & 0 & .033507 & -4.466802 & -.265767 \\ 9 & 6 & 0 & -.888205 & -5.145569 & .625819 \\ 10 & 1 & 0 & -.721271 & -6.207231 & .461947 \\ 11 & 1 & 0 & -1.914112 & -4.873898 & .372854 \\ 12 & 1 & 0 & -.667438 & -4.876478 & 1.659845 \\ 13 & 6 & 0 & -.000664 & .610152 & -.228689 \\ 14 & 6 & 0 & .000664 & -.610152 & -.228689 \\ 15 & 6 & 0 & -.000664 & -1.963710 & -.226800 \\ 16 & 6 & 0 & .001361 & -3.175905 & -.231748\end{array}$

- $\quad 1(3)\left(C_{2 \mathrm{~h}}\right)$

$\mathrm{HF}=-458.7913937$

NIMAG $=1$

\begin{tabular}{|c|c|c|c|c|c|}
\hline \multirow{2}{*}{$\begin{array}{l}\text { Center } \\
\text { Number }\end{array}$} & \multirow{2}{*}{\multicolumn{2}{|c|}{$\begin{array}{l}\text { Atomic } \\
\text { Number }\end{array}$}} & \multirow{2}{*}{$\begin{array}{l}\text { Atomic } \\
\text { Type }\end{array}$} & \multicolumn{2}{|c|}{ Coordinates (Angstroms } \\
\hline & & & & $x$ & Z \\
\hline 1 & 8 & 0 & .044053 & 4.468359 & .000000 \\
\hline 2 & 6 & 0 & -.000469 & 3.176316 & .000000 \\
\hline 3 & 6 & 0 & -1.239456 & 5.143254 & .000000 \\
\hline 4 & 1 & 0 & -1.008232 & 6.205460 & .000000 \\
\hline 5 & 1 & 0 & -1.800156 & 4.871935 & .895880 \\
\hline 6 & 1 & 0 & -1.800156 & 4.871935 & -.895880 \\
\hline 7 & 6 & 0 & -.003503 & 1.964771 & .000000 \\
\hline 8 & 8 & 0 & -.044053 & -4.468359 & .000000 \\
\hline 9 & 6 & 0 & 1.239456 & -5.143254 & .000000 \\
\hline 10 & 1 & 0 & 1.008232 & -6.205460 & .000000 \\
\hline 11 & 1 & 0 & 1.800156 & -4.871935 & .895880 \\
\hline 12 & 1 & 0 & 1.800156 & -4.871935 & -.895880 \\
\hline 13 & 6 & 0 & .000469 & .609758 & .000000 \\
\hline 14 & 6 & 0 & -.000469 & -.609758 & .000000 \\
\hline 15 & 6 & 0 & .003503 & -1.964771 & .000000 \\
\hline 16 & 6 & 0 & .000469 & -3.176316 & .000000 \\
\hline
\end{tabular}


- $\quad 1(4)\left(C_{2}\right)$

$H F=-534.9718683$

NIMAG $=0$

\begin{tabular}{|c|c|c|c|c|c|}
\hline \multirow{2}{*}{$\begin{array}{l}\text { Center } \\
\text { Number }\end{array}$} & \multirow{2}{*}{\multicolumn{2}{|c|}{$\begin{array}{l}\text { Atomic } \\
\text { Number }\end{array}$}} & \multirow{2}{*}{$\begin{array}{l}\text { Atomic } \\
\text { Type }\end{array}$} & \multicolumn{2}{|c|}{ Coordinates (Angstroms) } \\
\hline & & & & $Y$ & Z \\
\hline 1 & 8 & 0 & -.032838 & 5.747099 & -.231102 \\
\hline 2 & 6 & 0 & -.000560 & 4.458841 & -.197719 \\
\hline 3 & 6 & 0 & .887438 & 6.430982 & .661042 \\
\hline 4 & 1 & 0 & .717585 & 7.491264 & .492954 \\
\hline 5 & 1 & 0 & 1.913511 & 6.160002 & .409108 \\
\hline 6 & 1 & 0 & .664403 & 6.163683 & 1.694760 \\
\hline 7 & 6 & 0 & .000560 & 3.245619 & -.194833 \\
\hline 8 & 8 & 0 & .032838 & -5.747099 & -.231102 \\
\hline 9 & 6 & 0 & -.887438 & -6.430982 & .661042 \\
\hline 10 & 1 & 0 & -.717585 & -7.491264 & .492954 \\
\hline 11 & 1 & 0 & -1.913511 & -6.160002 & .409108 \\
\hline 12 & 1 & 0 & -.664403 & -6.163683 & 1.694760 \\
\hline 13 & 6 & 0 & -.001974 & 1.895650 & -.196939 \\
\hline 14 & 6 & 0 & -.000502 & .672034 & -.196218 \\
\hline 15 & 6 & 0 & .000502 & -.672034 & -.196218 \\
\hline 16 & 6 & 0 & .001974 & -1.895650 & -.196939 \\
\hline 17 & 6 & 0 & -.000560 & -3.245619 & -194833 \\
\hline 18 & 6 & 0 & .000560 & -4.458841 & -.197719 \\
\hline
\end{tabular}

- $\quad 1(4)\left(C_{2 h}\right)$

$\mathrm{HF}=-534.9714109$

NIMAG $=1$ 


\begin{tabular}{cccccc} 
Center & \multicolumn{2}{l}{ Atomic } & \multicolumn{2}{l}{ Atomic } & \multicolumn{3}{c}{ Coordinates (Angstroms) } \\
Number & Number & Type & X & $Y$ & $Z$ \\
& & & & & \\
1 & 8 & 0 & .042246 & 5.748271 & .000000 \\
2 & 6 & 0 & -.001708 & 4.459277 & .000000 \\
3 & 6 & 0 & -1.240777 & 6.428628 & .000000 \\
4 & 1 & 0 & -1.004460 & 7.489478 & .000000 \\
5 & 1 & 0 & -1.800835 & 6.158431 & .896251 \\
6 & 1 & 0 & -1.800835 & 6.158431 & -.896251 \\
7 & 6 & 0 & -.002949 & 3.246479 & .000000 \\
8 & 8 & 0 & -.042246 & -5.748271 & .000000 \\
9 & 6 & 0 & 1.240777 & -6.428628 & .000000 \\
10 & 1 & 0 & 1.004460 & -7.489478 & .000000 \\
11 & 1 & 0 & 1.800835 & -6.158431 & .896251 \\
12 & 1 & 0 & 1.800835 & -6.158431 & -.896251 \\
13 & 6 & 0 & .001708 & 1.895592 & .000000 \\
14 & 6 & 0 & .000239 & .672521 & .000000 \\
15 & 6 & 0 & -.000239 & -.672521 & .000000 \\
16 & 6 & 0 & -.001708 & -1.895592 & .000000 \\
17 & 6 & 0 & .002949 & -3.246479 & .000000 \\
18 & 6 & 0 & .001708 & -4.459277 & .000000
\end{tabular}

- $\quad 2(1)\left(C_{2}\right)$

$H F=-952.4329146$

NIMAG $=0$

\begin{tabular}{ccccccc} 
Center & \multicolumn{2}{c}{ Atomic } & \multicolumn{2}{c}{ Atomic } & \multicolumn{3}{c}{ Coordinates (Angstroms) } \\
Number & Number & Type & X & Y & Z \\
\hline 1 & 16 & 0 & -.017982 & 2.284304 & -.397286 \\
2 & 6 & 0 & .017982 & .609141 & -.328075 \\
3 & 6 & 0 & 1.255674 & 2.751485 & .846755 \\
4 & 1 & 0 & 1.287885 & 3.841743 & .854116 \\
5 & 1 & 0 & 2.228293 & 2.357683 & .557712
\end{tabular}




$\begin{array}{cccccc}6 & 1 & 0 & .974178 & 2.386095 & 1.832670 \\ 7 & 6 & 0 & -.017982 & -.609141 & -.328075 \\ 8 & 16 & 0 & .017982 & -2.284304 & -.397286 \\ 9 & 6 & 0 & -1.255674 & -2.751485 & .846755 \\ 10 & 1 & 0 & -1.287885 & -3.841743 & .854116 \\ 11 & 1 & 0 & -2.228293 & -2.357683 & .557712 \\ 12 & 1 & 0 & -.974178 & -2.386095 & 1.832670\end{array}$

- 2(1) $\left(C_{2 \mathrm{~h}}\right)$

$\mathrm{HF}=-952.425798$

$\mathrm{NIMAG}=1$

\begin{tabular}{cccccc} 
Center & \multicolumn{2}{c}{ Atomic } & \multicolumn{2}{c}{ Atomic } & \multicolumn{3}{c}{ Coordinates } \\
Number & Number & Type & X & Y & $Z$ \\
& & & & & \\
1 & 16 & 0 & .014678 & 2.304288 & .000000 \\
2 & 6 & 0 & -.014678 & .605940 & .000000 \\
3 & 6 & 0 & -1.786829 & 2.661704 & .000000 \\
4 & 1 & 0 & -1.876728 & 3.748248 & .000000 \\
5 & 1 & 0 & -2.254857 & 2.254894 & .894541 \\
6 & 1 & 0 & -2.254857 & 2.254894 & -.894541 \\
7 & 6 & 0 & .014678 & -.605940 & .000000 \\
8 & 16 & 0 & -.014678 & -2.304288 & .000000 \\
9 & 6 & 0 & 1.786829 & -2.661704 & .000000 \\
10 & 1 & 0 & 1.876728 & -3.748248 & .000000 \\
11 & 1 & 0 & 2.254857 & -2.254894 & .894541 \\
12 & 1 & 0 & 2.254857 & -2.254894 & -.894541
\end{tabular}

- $\quad 2(2)\left(C_{2}\right)$

$\mathrm{HF}=-1028.6106773$

$\mathrm{NIMAG}=0$ 


\begin{tabular}{|c|c|c|c|c|c|}
\hline \multirow{2}{*}{$\begin{array}{l}\text { Center } \\
\text { Number }\end{array}$} & \multirow{2}{*}{\multicolumn{2}{|c|}{$\begin{array}{l}\text { Atomic } \\
\text { Number }\end{array}$}} & \multirow{2}{*}{$\begin{array}{l}\text { Atomic } \\
\text { Type }\end{array}$} & \multicolumn{2}{|c|}{ Coordinates (Angstroms) } \\
\hline & & & & $\mathrm{Y}$ & Z \\
\hline 1 & 16 & 0 & -.063933 & 3.565628 & -.342953 \\
\hline 2 & 6 & 0 & .001601 & 1.895409 & -.279812 \\
\hline 3 & 6 & 0 & 1.199292 & 4.044485 & .908569 \\
\hline 4 & 1 & 0 & 1.212428 & 5.134851 & .921220 \\
\hline 5 & 1 & 0 & 2.178065 & 3.667485 & .618627 \\
\hline 6 & 1 & 0 & .918773 & 3.667887 & 1.890273 \\
\hline 7 & 6 & 0 & -.001601 & .673743 & -.285901 \\
\hline 8 & 16 & 0 & .063933 & -3.565628 & -.342953 \\
\hline 9 & 6 & 0 & -1.199292 & -4.044485 & .908569 \\
\hline 10 & 1 & 0 & -1.212428 & -5.134851 & .921220 \\
\hline 11 & 1 & 0 & -2.178065 & -3.667485 & .618627 \\
\hline 12 & 1 & 0 & -.918773 & -3.667887 & 1.890273 \\
\hline 13 & 6 & 0 & .001601 & -.673743 & -.285901 \\
\hline 14 & 6 & 0 & -.001601 & -1.895409 & -.279812 \\
\hline
\end{tabular}

- $\quad \mathbf{2 ( 2 )}\left(C_{2 \mathrm{~h}}\right)$

$H F=-1028.6066195$

$\mathrm{NIMAG}=1$

\begin{tabular}{|c|c|c|c|c|c|}
\hline Center & Atomic & \multirow{2}{*}{\multicolumn{2}{|c|}{$\begin{array}{l}\text { Atomic } \\
\text { Type }\end{array}$}} & \multicolumn{2}{|c|}{ Coordinates (Angstroms } \\
\hline Number & Number & & & $\mathrm{Y}$ & Z \\
\hline 1 & 16 & 0 & .073655 & 3.578490 & .000000 \\
\hline 2 & 6 & 0 & -.001010 & 1.895339 & .000000 \\
\hline 3 & 6 & 0 & -1.715418 & 4.005830 & .000000 \\
\hline 4 & 1 & 0 & -1.761435 & 5.095154 & .000000 \\
\hline 5 & 1 & 0 & -2.197179 & 3.617024 & .895001 \\
\hline 6 & 1 & 0 & -2.197179 & 3.617024 & -.895001 \\
\hline
\end{tabular}




$\begin{array}{cccccc}7 & 6 & 0 & .001010 & .677897 & .000000 \\ 8 & 16 & 0 & -.073655 & -3.578490 & .000000 \\ 9 & 6 & 0 & 1.715418 & -4.005830 & .000000 \\ 10 & 1 & 0 & 1.761435 & -5.095154 & .000000 \\ 11 & 1 & 0 & 2.197179 & -3.617024 & .895001 \\ 12 & 1 & 0 & 2.197179 & -3.617024 & -.895001 \\ 13 & 6 & 0 & -.001010 & -.677897 & .000000 \\ 14 & 6 & 0 & .001010 & -1.895339 & .000000\end{array}$

- $\quad 2(3)\left(C_{2}\right)$

$H F=-1104.7893658$

$\mathrm{NIMAG}=0$

\begin{tabular}{|c|c|c|c|c|}
\hline \multirow{2}{*}{$\begin{array}{l}\text { Center } \\
\text { Number }\end{array}$} & \multirow{2}{*}{$\begin{array}{l}\text { Atomic } \\
\text { Number }\end{array}$} & Atomic & \multicolumn{2}{|c|}{ Coordinates (Angstroms) } \\
\hline & & Type & $\mathrm{Y}$ & Z \\
\hline 1 & 16 & -.059491 & 4.847940 & -.301386 \\
\hline 2 & 6 & .001661 & 3.178627 & -.243642 \\
\hline 3 & 6 & 1.203037 & 5.315820 & .955307 \\
\hline 4 & 1 & 1.218901 & 6.405973 & .972391 \\
\hline 5 & 1 & 2.180843 & 4.936896 & .664972 \\
\hline 6 & 1 & .918367 & 4.935341 & 1.934190 \\
\hline 7 & 6 & -.003533 & 1.955615 & -.251039 \\
\hline 8 & 16 & .059491 & -4.847940 & -.301386 \\
\hline 9 & 6 & -1.203037 & -5.315820 & .955307 \\
\hline 10 & 1 & -1.218901 & -6.405973 & .972391 \\
\hline 11 & 1 & -2.180843 & -4.936896 & .664972 \\
\hline 12 & 1 & -.918367 & -4.935341 & 1.934190 \\
\hline 13 & 6 & -.001661 & .612955 & -.252190 \\
\hline 14 & 6 & .001661 & -.612955 & -.252190 \\
\hline 15 & 6 & .003533 & -1.955615 & -.251039 \\
\hline 16 & 6 & -.001661 & -3.178627 & -.243642 \\
\hline
\end{tabular}


- $\quad \mathbf{2 ( 3 )}\left(C_{2 \mathrm{~h}}\right)$

$H F=-1104.7868627$

NIMAG $=1$

\begin{tabular}{|c|c|c|c|c|c|}
\hline \multirow{2}{*}{$\begin{array}{l}\text { Center } \\
\text { Number }\end{array}$} & \multirow{2}{*}{\multicolumn{2}{|c|}{$\begin{array}{l}\text { Atomic } \\
\text { Number }\end{array}$}} & \multirow{2}{*}{$\begin{array}{l}\text { Atomic } \\
\text { Type }\end{array}$} & \multicolumn{2}{|c|}{ Coordinates (Angstroms } \\
\hline & & & & $\mathrm{Y}$ & Z \\
\hline 1 & 16 & 0 & .066892 & 4.856828 & .000000 \\
\hline 2 & 6 & 0 & -.008434 & 3.179569 & .000000 \\
\hline 3 & 6 & 0 & -1.720736 & 5.295176 & .000000 \\
\hline 4 & 1 & 0 & -1.759819 & 6.384757 & .000000 \\
\hline 5 & 1 & 0 & -2.203855 & 4.908823 & .895187 \\
\hline 6 & 1 & 0 & -2.203855 & 4.908823 & -.895187 \\
\hline 7 & 6 & 0 & -.000684 & 1.959344 & .000000 \\
\hline 8 & 16 & 0 & -.066892 & -4.856828 & .000000 \\
\hline 9 & 6 & 0 & 1.720736 & -5.295176 & .000000 \\
\hline 10 & 1 & 0 & 1.759819 & -6.384757 & .000000 \\
\hline 11 & 1 & 0 & 2.203855 & -4.908823 & .895187 \\
\hline 12 & 1 & 0 & 2.203855 & -4.908823 & -.895187 \\
\hline 13 & 6 & 0 & .000684 & .611439 & .000000 \\
\hline 14 & 6 & 0 & -.000684 & -.611439 & .000000 \\
\hline 15 & 6 & 0 & .000684 & -1.959344 & .000000 \\
\hline 16 & 6 & 0 & .008434 & -3.179569 & .000000 \\
\hline
\end{tabular}

- $\quad 2(4)\left(C_{2}\right)$

$H F=-1180.9682628$

$\mathrm{NIMAG}=0$

\begin{tabular}{|c|c|c|c|c|}
\hline Center & Atomic & Atomic & Coordinates & (Angstroms \\
\hline Number & Number & Type & $x$ & Z \\
\hline 1 & 16 & -.057887 & 6.129705 & -.268584 \\
\hline 2 & 6 & .001307 & 4.460702 & -.213132 \\
\hline
\end{tabular}




\begin{tabular}{|c|c|c|c|c|c|}
\hline 3 & 6 & 0 & 1.206486 & 6.591993 & .988497 \\
\hline 4 & 1 & 0 & 1.223534 & 7.682008 & 1.007617 \\
\hline 5 & 1 & 0 & 2.183338 & 6.212304 & .696185 \\
\hline 6 & 1 & 0 & .921453 & 6.209727 & 1.966502 \\
\hline 7 & 6 & 0 & -.005052 & 3.237227 & -.222042 \\
\hline 8 & 16 & 0 & .057887 & -6.129705 & -.268584 \\
\hline 9 & 6 & 0 & -1.206486 & -6.591993 & .988497 \\
\hline 10 & 1 & 0 & -1.223534 & -7.682008 & 1.007617 \\
\hline 11 & 1 & 0 & -2.183338 & -6.212304 & .696185 \\
\hline 12 & 1 & 0 & -.921453 & -6.209727 & 1.966502 \\
\hline 13 & 6 & 0 & -.003959 & 1.896281 & -.224266 \\
\hline 14 & 6 & 0 & -.001307 & .668447 & -.224550 \\
\hline 15 & 6 & 0 & .001307 & -.668447 & -.224550 \\
\hline 16 & 6 & 0 & .003959 & -1.896281 & -.224266 \\
\hline 17 & 6 & 0 & .005052 & -3.237227 & -.222042 \\
\hline 18 & 6 & 0 & -.001307 & -4.460702 & -.213132 \\
\hline
\end{tabular}

\footnotetext{
- $\quad 2(4)\left(C_{2 h}\right)$

$H F=-1180.9666594$

$\mathrm{NIMAG}=1$
}

\begin{tabular}{|c|c|c|c|c|c|}
\hline \multirow{2}{*}{$\begin{array}{l}\text { Center } \\
\text { Number }\end{array}$} & \multirow{2}{*}{\multicolumn{2}{|c|}{$\begin{array}{l}\text { Atomic } \\
\text { Number }\end{array}$}} & & \multicolumn{2}{|c|}{ Coordinates (Angstroms } \\
\hline & & & & $\mathrm{Y}$ & Z \\
\hline 1 & 16 & 0 & .070253 & 6.135823 & .000000 \\
\hline 2 & 6 & 0 & -.008738 & 4.461927 & .000000 \\
\hline 3 & 6 & 0 & -1.715757 & 6.583673 & .000000 \\
\hline 4 & 1 & 0 & -1.748601 & 7.673427 & .000000 \\
\hline 5 & 1 & 0 & -2.200274 & 6.199700 & .895359 \\
\hline 6 & 1 & 0 & -2.200274 & 6.199700 & -.895359 \\
\hline 7 & 6 & 0 & -.000103 & 3.240309 & .000000 \\
\hline 8 & 16 & 0 & -.070253 & -6.135823 & .000000 \\
\hline 9 & 6 & 0 & 1.715757 & -6.583673 & .000000 \\
\hline 10 & 1 & 0 & 1.748601 & -7.673427 & .000000 \\
\hline 11 & 1 & 0 & 2.200274 & -6.199700 & .895359 \\
\hline
\end{tabular}




$\begin{array}{llllll}12 & 1 & 0 & 2.200274 & -6.199700 & -.895359 \\ 13 & 6 & 0 & .001138 & 1.895913 & .000000 \\ 14 & 6 & 0 & .000103 & .670220 & .000000 \\ 15 & 6 & 0 & -.000103 & -.670220 & .000000 \\ 16 & 6 & 0 & -.001138 & -1.895913 & .000000 \\ 17 & 6 & 0 & .000103 & -3.240309 & .000000 \\ 18 & 6 & 0 & .008738 & -4.461927 & .000000\end{array}$

- $3(1)\left(C_{2}\right)$

$\mathrm{HF}=-4959.0969232$

$\mathrm{NIMAG}=0$

\begin{tabular}{|c|c|c|c|c|c|}
\hline \multirow{2}{*}{$\begin{array}{l}\text { Center } \\
\text { Number }\end{array}$} & \multirow{2}{*}{\multicolumn{2}{|c|}{$\begin{array}{l}\text { Atomic } \\
\text { Number }\end{array}$}} & \multirow{2}{*}{$\begin{array}{l}\text { Atomic } \\
\text { Type }\end{array}$} & \multicolumn{2}{|c|}{ Coordinates (Angstroms } \\
\hline & & & & $\mathrm{Y}$ & Z \\
\hline 1 & 34 & 0 & -.021359 & 2.431738 & -.273693 \\
\hline 2 & 6 & 0 & .021359 & .608865 & -.191211 \\
\hline 3 & 6 & 0 & 1.364718 & 2.842141 & 1.083764 \\
\hline 4 & 1 & 0 & 1.439132 & 3.929129 & 1.123340 \\
\hline 5 & 1 & 0 & 2.314875 & 2.412618 & .776074 \\
\hline 6 & 1 & 0 & 1.056707 & 2.452264 & 2.050831 \\
\hline 7 & 6 & 0 & -.021359 & -.608865 & -.191211 \\
\hline 8 & 34 & 0 & .021359 & -2.431738 & -.273693 \\
\hline 9 & 6 & 0 & -1.364718 & -2.842141 & 1.083764 \\
\hline 10 & 1 & 0 & -1.439132 & -3.929129 & 1.123340 \\
\hline 11 & 1 & 0 & -2.314875 & -2.412618 & .776074 \\
\hline 12 & 1 & 0 & -1.056707 & -2.452264 & 2.050831 \\
\hline
\end{tabular}

- $\quad 3(1)\left(C_{2 h}\right)$

$\mathrm{HF}=-4959.0917068$

$\mathrm{NIMAG}=1$ 


\begin{tabular}{|c|c|c|c|c|c|}
\hline \multirow{2}{*}{$\begin{array}{l}\text { Center } \\
\text { Number }\end{array}$} & \multirow{2}{*}{\multicolumn{2}{|c|}{$\begin{array}{l}\text { Atomic } \\
\text { Number }\end{array}$}} & \multirow{2}{*}{$\begin{array}{l}\text { Atomic } \\
\text { Type }\end{array}$} & \multicolumn{2}{|c|}{ Coordinates (Angstroms } \\
\hline & & & & $\mathrm{Y}$ & Z \\
\hline 1 & 34 & 0 & .018985 & 2.449474 & .000000 \\
\hline 2 & 6 & 0 & -.018985 & .606610 & .000000 \\
\hline 3 & 6 & 0 & -1.937640 & 2.750197 & .000000 \\
\hline 4 & 1 & 0 & -2.075297 & 3.831315 & .000000 \\
\hline 5 & 1 & 0 & -2.375100 & 2.317145 & .896156 \\
\hline 6 & 1 & 0 & -2.375100 & 2.317145 & -.896156 \\
\hline 7 & 6 & 0 & .018985 & -.606610 & .000000 \\
\hline 8 & 34 & 0 & -.018985 & -2.449474 & .000000 \\
\hline 9 & 6 & 0 & 1.937640 & -2.750197 & .000000 \\
\hline 10 & 1 & 0 & 2.075297 & -3.831315 & .000000 \\
\hline 11 & 1 & 0 & 2.375100 & -2.317145 & .896156 \\
\hline 12 & 1 & 0 & 2.375100 & -2.317145 & -.896156 \\
\hline
\end{tabular}

- $\quad 3(2)\left(C_{2}\right)$

$H F=-5035.2742938$

$\mathrm{NIMAG}=0$

\begin{tabular}{|c|c|c|c|c|c|}
\hline \multirow{2}{*}{$\begin{array}{l}\text { Center } \\
\text { Number }\end{array}$} & \multirow{2}{*}{\multicolumn{2}{|c|}{$\begin{array}{l}\text { Atomic } \\
\text { Number }\end{array}$}} & \multirow{2}{*}{$\begin{array}{l}\text { Atomic } \\
\text { Type }\end{array}$} & \multicolumn{2}{|c|}{ Coordinates (Angstroms } \\
\hline & & & & $\mathrm{Y}$ & Z \\
\hline 1 & 34 & 0 & -.079998 & 3.712088 & -.252357 \\
\hline 2 & 6 & 0 & .002561 & 1.896493 & -.173035 \\
\hline 3 & 6 & 0 & 1.293776 & 4.145286 & 1.111954 \\
\hline 4 & 1 & 0 & 1.340598 & 5.233374 & 1.157780 \\
\hline 5 & 1 & 0 & 2.253882 & 3.740202 & .802469 \\
\hline 6 & 1 & 0 & .991048 & 3.740950 & 2.074547 \\
\hline 7 & 6 & 0 & -.002561 & .675419 & -.181363 \\
\hline 8 & 34 & 0 & .079998 & -3.712088 & -.252357 \\
\hline 9 & 6 & 0 & -1.293776 & -4.145286 & 1.111954 \\
\hline
\end{tabular}




$\begin{array}{llllll}10 & 1 & 0 & -1.340598 & -5.233374 & 1.157780 \\ 11 & 1 & 0 & -2.253882 & -3.740202 & .802469 \\ 12 & 1 & 0 & -.991048 & -3.740950 & 2.074547 \\ 13 & 6 & 0 & .002561 & -.675419 & -.181363 \\ 14 & 6 & 0 & -.002561 & -1.896493 & -.173035\end{array}$

- $3(2)\left(C_{2 h}\right)$

$H F=-5035.2712348$

NIMAG $=1$

\begin{tabular}{|c|c|c|c|c|c|}
\hline \multirow{2}{*}{$\begin{array}{l}\text { Center } \\
\text { Number }\end{array}$} & \multirow{2}{*}{$\begin{array}{l}\text { Atomic } \\
\text { Number }\end{array}$} & \multirow{2}{*}{\multicolumn{2}{|c|}{$\begin{array}{l}\text { Atomic } \\
\text { Type }\end{array}$}} & \multicolumn{2}{|c|}{ Coordinates (Angstroms } \\
\hline & & & & $\mathrm{Y}$ & Z \\
\hline 1 & 34 & 0 & .088974 & 3.724070 & .000000 \\
\hline 2 & 6 & 0 & -.002531 & 1.896441 & .000000 \\
\hline 3 & 6 & 0 & -1.856964 & 4.100962 & .000000 \\
\hline 4 & 1 & 0 & -1.951314 & 5.186748 & .000000 \\
\hline 5 & 1 & 0 & -2.309069 & 3.684343 & .896480 \\
\hline 6 & 1 & 0 & -2.309069 & 3.684343 & -.896480 \\
\hline 7 & 6 & 0 & .002531 & .678527 & .000000 \\
\hline 8 & 34 & 0 & -.088974 & -3.724070 & .000000 \\
\hline 9 & 6 & 0 & 1.856964 & -4.100962 & .000000 \\
\hline 10 & 1 & 0 & 1.951314 & -5.186748 & .000000 \\
\hline 11 & 1 & 0 & 2.309069 & -3.684343 & .896480 \\
\hline 12 & 1 & 0 & 2.309069 & -3.684343 & -.896480 \\
\hline 13 & 6 & 0 & -.002531 & -.678527 & .000000 \\
\hline 14 & 6 & 0 & .002531 & -1.896441 & .000000 \\
\hline
\end{tabular}

- $3(3)\left(C_{2}\right)$

$H F=-5111.4530942$

$\mathrm{NIMAG}=0$ 


\begin{tabular}{|c|c|c|c|c|c|}
\hline \multirow{2}{*}{$\begin{array}{l}\text { Center } \\
\text { Number }\end{array}$} & \multirow{2}{*}{\multicolumn{2}{|c|}{$\begin{array}{l}\text { Atomic } \\
\text { Number }\end{array}$}} & \multirow{2}{*}{$\begin{array}{l}\text { Atomic } \\
\text { Type }\end{array}$} & \multicolumn{2}{|c|}{ Coordinates (Angstroms) } \\
\hline & & & & $\mathrm{Y}$ & Z \\
\hline 1 & 34 & 0 & -.075010 & 4.994843 & -.233139 \\
\hline 2 & 6 & 0 & .001569 & 3.179998 & -.159978 \\
\hline 3 & 6 & 0 & 1.299247 & 5.414831 & 1.134951 \\
\hline 4 & 1 & 0 & 1.349245 & 6.502451 & 1.185706 \\
\hline 5 & 1 & 0 & 2.257860 & 5.007760 & .823743 \\
\hline 6 & 1 & 0 & .993216 & 5.006566 & 2.094730 \\
\hline 7 & 6 & 0 & -.004351 & 1.957331 & -.168568 \\
\hline 8 & 34 & 0 & .075010 & -4.994843 & -.233139 \\
\hline 9 & 6 & 0 & -1.299247 & -5.414831 & 1.134951 \\
\hline 10 & 1 & 0 & -1.349245 & -6.502451 & 1.185706 \\
\hline 11 & 1 & 0 & -2.257860 & -5.007760 & .823743 \\
\hline 12 & 1 & 0 & -.993216 & -5.006566 & 2.094730 \\
\hline 13 & 6 & 0 & -.001569 & .612460 & -.169311 \\
\hline 14 & 6 & 0 & .001569 & -.612460 & -.169311 \\
\hline 15 & 6 & 0 & .004351 & -1.957331 & -.168568 \\
\hline 16 & 6 & 0 & -.001569 & -3.179998 & -.159978 \\
\hline
\end{tabular}

- $3(3)\left(C_{2 \mathrm{~h}}\right)$

$H F=-5111.4511887$

$\mathrm{NIMAG}=1$

\begin{tabular}{|c|c|c|c|c|c|}
\hline Center & Atomic & \multirow{2}{*}{\multicolumn{2}{|c|}{$\begin{array}{l}\text { Atomic } \\
\text { Type }\end{array}$}} & \multicolumn{2}{|c|}{ Coordinates (Angstroms } \\
\hline Number & Numbel & & & $x$ & Z \\
\hline 1 & 34 & 0 & .077968 & 5.003079 & .000000 \\
\hline 2 & 6 & 0 & -.010806 & 3.180700 & .000000 \\
\hline 3 & 6 & 0 & -1.867995 & 5.385300 & .000000 \\
\hline 4 & 1 & 0 & -1.958800 & 6.471363 & .000000 \\
\hline 5 & 1 & 0 & -2.320375 & 4.969613 & .896672 \\
\hline
\end{tabular}




$\begin{array}{cccccc}6 & 1 & 0 & -2.320375 & 4.969613 & -.896672 \\ 7 & 6 & 0 & -.000380 & 1.960130 & .000000 \\ 8 & 34 & 0 & -.077968 & -5.003079 & .000000 \\ 9 & 6 & 0 & 1.867995 & -5.385300 & .000000 \\ 10 & 1 & 0 & 1.958800 & -6.471363 & .000000 \\ 11 & 1 & 0 & 2.320375 & -4.969613 & .896672 \\ 12 & 1 & 0 & 2.320375 & -4.969613 & -.896672 \\ 13 & 6 & 0 & .000380 & .611330 & .000000 \\ 14 & 6 & 0 & -.000380 & -.611330 & .000000 \\ 15 & 6 & 0 & .000380 & -1.960130 & .000000 \\ 16 & 6 & 0 & .010806 & -3.180700 & .000000\end{array}$

- $3(4)\left(C_{2}\right)$

$\mathrm{HF}=-5187.6320507$

$\mathrm{NIMAG}=0$

\begin{tabular}{|c|c|c|c|c|c|}
\hline \multirow{2}{*}{$\begin{array}{l}\text { Center } \\
\text { Number }\end{array}$} & \multirow{2}{*}{\multicolumn{2}{|c|}{$\begin{array}{l}\text { Atomic } \\
\text { Number }\end{array}$}} & Atomic & \multicolumn{2}{|c|}{ Coordinates (Angstroms } \\
\hline & & & Type & $x$ & Z \\
\hline 1 & 34 & 0 & -.072513 & 6.276749 & -.216799 \\
\hline 2 & 6 & 0 & .001378 & 4.462213 & -.146310 \\
\hline 3 & 6 & 0 & 1.303129 & 6.690265 & 1.151929 \\
\hline 4 & 1 & 0 & 1.354703 & 7.777650 & 1.204616 \\
\hline 5 & 1 & 0 & 2.260727 & 6.282146 & .839142 \\
\hline 6 & 1 & 0 & .996175 & 6.280495 & 2.110713 \\
\hline 7 & 6 & 0 & -.005538 & 3.238934 & -.155590 \\
\hline 8 & 34 & 0 & .072513 & -6.276749 & -.216799 \\
\hline 9 & 6 & 0 & -1.303129 & -6.690265 & 1.151929 \\
\hline 10 & 1 & 0 & -1.354703 & -7.777650 & 1.204616 \\
\hline 11 & 1 & 0 & -2.260727 & -6.282146 & .839142 \\
\hline 12 & 1 & 0 & -.996175 & -6.280495 & 2.110713 \\
\hline 13 & 6 & 0 & -.004021 & 1.896078 & -.156861 \\
\hline 14 & 6 & 0 & -.001378 & .668968 & -.157051 \\
\hline 15 & 6 & 0 & .001378 & -.668968 & -.157051 \\
\hline
\end{tabular}




$\begin{array}{llllll}16 & 6 & 0 & .004021 & -1.896078 & -.156861 \\ 17 & 6 & 0 & .005538 & -3.238934 & -.155590 \\ 18 & 6 & 0 & -.001378 & -4.462213 & -.146310\end{array}$

- $\quad 3(4)\left(C_{2 h}\right)$

$H F=-5187.6308154$

$\mathrm{NIMAG}=1$

\begin{tabular}{|c|c|c|c|c|c|}
\hline \multirow{2}{*}{$\begin{array}{l}\text { Center } \\
\text { Number }\end{array}$} & \multirow{2}{*}{\multicolumn{2}{|c|}{$\begin{array}{l}\text { Atomic } \\
\text { Number }\end{array}$}} & \multirow{2}{*}{$\begin{array}{l}\text { Atomic } \\
\text { Type }\end{array}$} & \multicolumn{2}{|c|}{ Coordinates (Angstroms } \\
\hline & & & & $\mathrm{Y}$ & Z \\
\hline 1 & 34 & 0 & .079993 & 6.282491 & .000000 \\
\hline 2 & 6 & 0 & -.012149 & 4.463144 & .000000 \\
\hline 3 & 6 & 0 & -1.864805 & 6.673013 & .000000 \\
\hline 4 & 1 & 0 & -1.950421 & 7.759477 & .000000 \\
\hline 5 & 1 & 0 & -2.318553 & 6.259250 & .896813 \\
\hline 6 & 1 & 0 & -2.318553 & 6.259250 & -.896813 \\
\hline 7 & 6 & 0 & -.000651 & 3.241272 & .000000 \\
\hline 8 & 34 & 0 & -.079993 & -6.282491 & .000000 \\
\hline 9 & 6 & 0 & 1.864805 & -6.673013 & .000000 \\
\hline 10 & 1 & 0 & 1.950421 & -7.759477 & .000000 \\
\hline 11 & 1 & 0 & 2.318553 & -6.259250 & .896813 \\
\hline 12 & 1 & 0 & 2.318553 & -6.259250 & -.896813 \\
\hline 13 & 6 & 0 & .000651 & 1.895816 & .000000 \\
\hline 14 & 6 & 0 & .000078 & .670297 & .000000 \\
\hline 15 & 6 & 0 & -.000078 & -.670297 & .000000 \\
\hline 16 & 6 & 0 & -.000651 & -1.895816 & .000000 \\
\hline 17 & 6 & 0 & .000651 & -3.241272 & .000000 \\
\hline 18 & 6 & 0 & .012149 & -4.463144 & .000000 \\
\hline
\end{tabular}


4.) He(II) Photoelectron Spectra of 2(2) (top) and 2(3) (bottom).
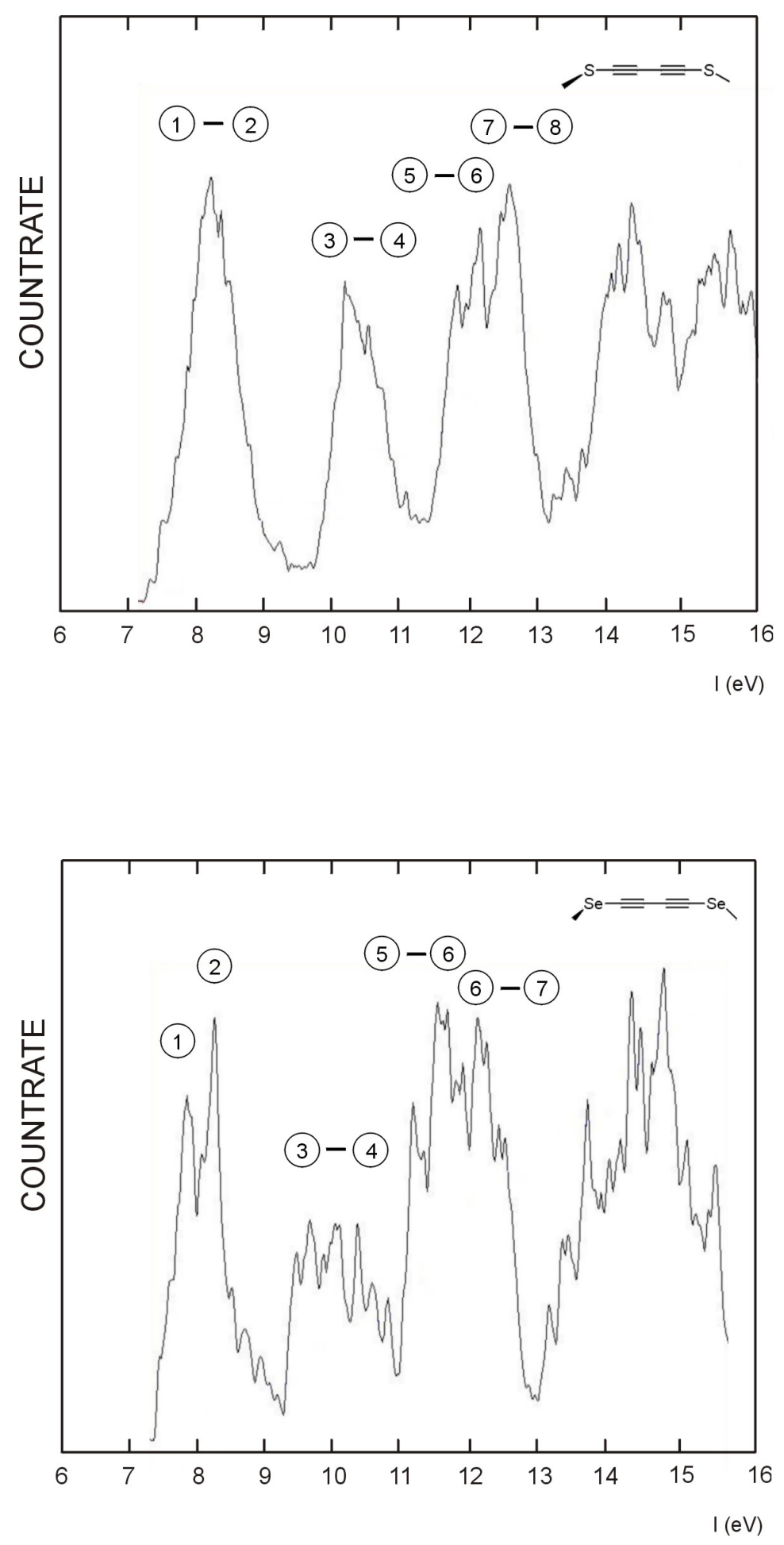


\section{5.) X-ray Data and Details of the Refinement Procedure for 3(4).}

Table 1: Crystal data and structure refinement for 3(4).

\begin{tabular}{|c|c|c|}
\hline Empirical formula & \multicolumn{2}{|l|}{$\mathrm{C}_{10} \mathrm{H}_{6} \mathrm{Se}_{2}$} \\
\hline Formula weight & \multicolumn{2}{|l|}{284.07} \\
\hline Temperature & \multicolumn{2}{|l|}{$100(2) \mathrm{K}$} \\
\hline Wavelength & \multicolumn{2}{|l|}{$0.71073 \AA$} \\
\hline Crystal system & \multicolumn{2}{|l|}{ Orthorhombic } \\
\hline Space group & \multicolumn{2}{|l|}{ Pbcn } \\
\hline \multirow[t]{3}{*}{ Unit cell dimensions } & $a=15.596(4) \AA$ & $\alpha=90 \mathrm{deg}$ \\
\hline & $\mathrm{b}=4.3034(11) \AA$ & $\beta=90 \mathrm{deg}$ \\
\hline & $c=28.359(7) \AA$ & $\gamma=90 \mathrm{deg}$ \\
\hline Volume & \multicolumn{2}{|l|}{$1903.4(8) \AA^{3}$} \\
\hline Density (calculated) & \multicolumn{2}{|l|}{$1.98 \mathrm{~g} / \mathrm{cm}^{3}$} \\
\hline Absorption coefficient & \multicolumn{2}{|l|}{$7.71 \mathrm{~mm}^{-1}$} \\
\hline Crystal shape & \multicolumn{2}{|l|}{ polyhedron } \\
\hline Crystal size & \multicolumn{2}{|c|}{$0.16 \times 0.11 \times 0.07 \mathrm{~mm}^{3}$} \\
\hline Theta range for data collection & \multicolumn{2}{|l|}{1.4 to $23.2 \mathrm{deg}$. } \\
\hline Index ranges & \multicolumn{2}{|c|}{$-17 \leq h \leq 17,-4 \leq k \leq 4,-31 \leq 1 \leq 31$} \\
\hline Reflections collected & \multicolumn{2}{|l|}{11002} \\
\hline Independent reflections & \multicolumn{2}{|c|}{$1370(\mathrm{R}$ (int) $=0.1159)$} \\
\hline Observed reflections & \multicolumn{2}{|l|}{$738(\mathrm{I}>2 \sigma(\mathrm{I}))$} \\
\hline Absorption correction & \multicolumn{2}{|c|}{ Semi-empirical from equivalents } \\
\hline Max. and min. transmission & \multicolumn{2}{|c|}{0.61 and 0.37} \\
\hline Refinement method & \multicolumn{2}{|c|}{ Full-matrix least-squares on $\mathrm{F}^{2}$} \\
\hline Data/restraints/parameters & \multirow{2}{*}{\multicolumn{2}{|c|}{$1370 / 0 / 45$}} \\
\hline Goodness-of-fit on $\mathrm{F}^{2}$ & & \\
\hline Final $R$ indices $(I>2 \sigma(I))$ & \multicolumn{2}{|c|}{$R 1=0.099, w R 2=0.240$} \\
\hline Largest diff. peak and hole & \multicolumn{2}{|l|}{8.61 and $-1.26 \mathrm{e}^{-3}$} \\
\hline
\end{tabular}

Table 2: Atomic coordinates and equivalent isotropic displacement parameters $\left(\AA^{2}\right)$ for $3(4)$. $U_{\text {eq }}$ is defined as one third of the trace of the orthogonalized $U_{i j}$ tensor.

\begin{tabular}{lllll}
\hline Atom & $\mathrm{x}$ & $\mathrm{y}$ & $\mathrm{z}$ & $\mathrm{U}_{\text {eq }}$ \\
\hline Se1 & $0.6523(1)$ & $1.4206(4)$ & $0.4268(1)$ & $0.0059(7)$ \\
Se2 & $0.6528(1)$ & $0.0861(4)$ & $0.8214(1)$ & $0.0059(7)$ \\
C1 & $0.6535(10)$ & $1.232(4)$ & $0.4840(5)$ & $0.0059(7)$ \\
C2 & $0.6549(11)$ & $1.104(4)$ & $0.5205(5)$ & $0.0059(7)$ \\
C3 & $0.6575(14)$ & $0.956(4)$ & $0.5648(6)$ & $0.0059(7)$ \\
C4 & $0.6568(10)$ & $0.826(4)$ & $0.6042(5)$ & $0.0059(7)$ \\
C5 & $0.6578(10)$ & $0.671(4)$ & $0.6444(5)$ & $0.0059(7)$ \\
C6 & $0.6576(14)$ & $0.538(4)$ & $0.6834(6)$ & $0.0059(7)$ \\
C7 & $0.6562(11)$ & $0.411(4)$ & $0.7275(5)$ & $0.0059(7)$ \\
C8 & $0.6546(10)$ & $0.272(4)$ & $0.7637(5)$ & $0.0059(7)$ \\
C11 & $0.5375(11)$ & $1.604(4)$ & $0.4334(5)$ & $0.0059(7)$ \\
C12 & $0.5380(11)$ & $-0.099(4)$ & $0.8169(5)$ & $0.0059(7)$ \\
\hline
\end{tabular}


Table 3: Hydrogen coordinates and isotropic displacement parameters $\left(\hat{A}^{2}\right)$ for 3(4).

\begin{tabular}{lllll}
\hline Atom & $x$ & $y$ & $z$ & $U_{\text {eq }}$ \\
\hline H11A & 0.4967 & 1.4419 & 0.4427 & 0.009 \\
H11B & 0.5198 & 1.6947 & 0.4033 & 0.009 \\
H11C & 0.5389 & 1.7661 & 0.4577 & 0.009 \\
H12A & 0.4963 & 0.0632 & 0.8089 & 0.009 \\
H12B & 0.5227 & -0.1922 & 0.8473 & 0.009 \\
H12C & 0.5379 & -0.2588 & 0.7924 & 0.009 \\
\hline
\end{tabular}

Table 4: $\quad$ Anisotropic displacement parameters $\left(\AA^{2}\right)$ for 3(4). The anisotropic displacement factor exponent takes the form: $-2 \mathrm{pi}^{2}\left(\mathrm{~h}^{2} \mathrm{a}^{* 2} \mathrm{U}_{11}+\ldots+2 \mathrm{hk} \mathrm{a}^{*} \mathrm{~b}^{*}\right.$ $\mathrm{U}_{12}$ )

\begin{tabular}{lllllll}
\hline Atom & $U_{11}$ & $U_{22}$ & $U_{33}$ & $U_{23}$ & $U_{13}$ & $U_{12}$ \\
\hline Se1 & $0.0035(9)$ & $0.0134(10)$ & $0.0009(9)$ & $0.0015(4)$ & $0.0006(5)$ & $0.0003(8)$ \\
Se2 & $0.0035(9)$ & $0.0134(10)$ & $0.0009(9)$ & $0.0015(4)$ & $0.0006(5)$ & $0.0003(8)$ \\
C1 & $0.0035(9)$ & $0.0134(10)$ & $0.0009(9)$ & $0.0015(4)$ & $0.0006(5)$ & $0.0003(8)$ \\
C2 & $0.0035(9)$ & $0.0134(10)$ & $0.0009(9)$ & $0.0015(4)$ & $0.0006(5)$ & $0.0003(8)$ \\
C3 & $0.0035(9)$ & $0.0134(10)$ & $0.0009(9)$ & $0.0015(4)$ & $0.0006(5)$ & $0.0003(8)$ \\
C4 & $0.0035(9)$ & $0.0134(10)$ & $0.0009(9)$ & $0.0015(4)$ & $0.0006(5)$ & $0.0003(8)$ \\
C5 & $0.0035(9)$ & $0.0134(10)$ & $0.0009(9)$ & $0.0015(4)$ & $0.0006(5)$ & $0.0003(8)$ \\
C6 & $0.0035(9)$ & $0.0134(10)$ & $0.0009(9)$ & $0.0015(4)$ & $0.0006(5)$ & $0.0003(8)$ \\
C7 & $0.0035(9)$ & $0.0134(10)$ & $0.0009(9)$ & $0.0015(4)$ & $0.0006(5)$ & $0.0003(8)$ \\
C8 & $0.0035(9)$ & $0.0134(10)$ & $0.0009(9)$ & $0.0015(4)$ & $0.0006(5)$ & $0.0003(8)$ \\
C11 & $0.0035(9)$ & $0.0134(10)$ & $0.0009(9)$ & $0.0015(4)$ & $0.0006(5)$ & $0.0003(8)$ \\
C12 & $0.0035(9)$ & $0.0134(10)$ & $0.0009(9)$ & $0.0015(4)$ & $0.0006(5)$ & $0.0003(8)$ \\
\hline
\end{tabular}

Table 5: $\quad$ Bond lengths $(\AA ̊)$ and angles (deg) for 3(4).

\begin{tabular}{lcll}
\hline Se1-C1 & $1.817(16)$ & C4-C3-C2 & $178(2)$ \\
Se1-C11 & $1.965(17)$ & C3-C4-C5 & $176.3(19)$ \\
Se2-C8 & $1.819(16)$ & C6-C5-C4 & $176.9(19)$ \\
Se2-C12 & $1.962(18)$ & C5-C6-C7 & $176(2)$ \\
C1-C2 & $1.17(2)$ & C8-C7-C6 & $173(2)$ \\
C2-C3 & $1.41(2)$ & C7-C8-Se2 & $175.8(17)$ \\
C3-C4 & $1.25(2)$ & Se1-C11-H11A & 109.5 \\
C4-C5 & $1.32(2)$ & Se1-C11-H11B & 109.5 \\
C5-C6 & $1.24(2)$ & H11A-C11-H11B & 109.5 \\
C6-C7 & $1.37(2)$ & Se1-C11-H11C & 109.5 \\
C7-C8 & $1.19(2)$ & H11A-C11-H11C & 109.5 \\
C11-H11A & 0.9800 & H11B-C11-H11C & 109.5 \\
C11-H11B & 0.9800 & Se2-C12-H12A & 109.5 \\
C11-H11C & 0.9800 & H12A-C12-H12B & 109.5 \\
C12-H12A & 0.9800 & Se2-C12-H12C & 109.5 \\
C12-H12B & 0.9800 & H12-C12-H12C & 109.5 \\
C12-H12C & 0.9800 & & \\
C1-Se1-C11 & $95.9(7)$ & & \\
C8-Se2-C12 & $97.7(7)$ & & \\
C2-C1-Se1 & $178.5(18)$ & & \\
C1-C2-C3 & $179(2)$ & & \\
& & &
\end{tabular}


